\title{
A New Spin on Galactic Dust
}

\section{Citation}

De Oliveira\#Costa, Angelica, Max Tegmark, Douglas P. Finkbeiner, R. D. Davies, Carlos M. Gutierrez, L. M. Haffner, Aled W. Jones, et al. 2002. "A New Spin on Galactic Dust." The Astrophysical Journal 567 (1) (March): 363-369. doi:10.1086/338109.

\section{Published Version}

doi:10.1086/338109

\section{Permanent link}

http://nrs.harvard.edu/urn-3:HUL.InstRepos:33462898

\section{Terms of Use}

This article was downloaded from Harvard University's DASH repository, and is made available under the terms and conditions applicable to Other Posted Material, as set forth at http:// nrs.harvard.edu/urn-3:HUL.InstRepos:dash.current.terms-of-use\#LAA

\section{Share Your Story}

The Harvard community has made this article openly available.

Please share how this access benefits you. Submit a story.

Accessibility 
The Astrophysical JouRnAL, 567:363-369, 2002 March 1

\title{
A NEW SPIN ON GALACTIC DUST
}

\author{
Angélica de Oliveira-Costa, ${ }^{1}$ Max Tegmark, ${ }^{1}$ Douglas P. Finkbeiner, ${ }^{2}$ R. D. Davies, ${ }^{3}$ Carlos M. Gutierrez, ${ }^{4}$ \\ L. M. Haffner, ${ }^{5}$ Aled W. Jones, ${ }^{6}$ A. N. Lasenby, ${ }^{6}$ R. Rebolo, ${ }^{4}$ Ron J. Reynolds, ${ }^{5}$ \\ S. L. TUFTE, ${ }^{7}$ AND R. A. WATSON ${ }^{3,4}$ \\ Received 2000 October 28; accepted 2001 October 16
}

\begin{abstract}
We present a new puzzle involving Galactic microwave emission and attempt to resolve it. On one hand, a cross-correlation analysis of the Wisconsin $\mathrm{H} \alpha$ Mapper map with the Tenerife 10 and $15 \mathrm{GHz}$ maps shows that the well-known DIRBE correlated microwave emission cannot be dominated by freefree emission. On the other hand, recent high-resolution observations in the $8-10 \mathrm{GHz}$ range with the Green Bank 140 foot telescope by Finkbeiner et al. failed to find the corresponding $8 \sigma$ signal that would be expected in the simplest spinning-dust models. So what physical mechanism is causing this ubiquitous dust-correlated emission? We argue for a model predicting that spinning dust is the culprit after all, but that the corresponding small grains are well correlated with the larger grains seen at 100 $\mu \mathrm{m}$ only on large angular scales. In support of this grain-segregation model, we find that the best spinning-dust template involves higher frequency maps in the range 12-60 $\mu \mathrm{m}$, in which emission from transiently heated small grains is important. Upcoming cosmic microwave background experiments such as ground-based interferometers, the Microwave Anisotropy Probe, and the Planck low-frequency interferometer with high resolution at low frequencies should allow a definitive test of this model.

Subject headings: cosmic microwave background - dust, extinction - infrared: ISM -

methods: data analysis - radiation mechanisms: nonthermal -

radiation mechanisms: thermal
\end{abstract}

\section{INTRODUCTION}

Understanding the physical origin of Galactic microwave emission is interesting for two reasons: to determine the fundamental properties of the Galactic components and to refine the modeling of foreground emission in cosmic microwave background (CMB) experiments. There are three Galactic foregrounds currently identified: synchrotron radiation, free-free emission, and thermal (vibrational) emission from dust grains. In the last few years, however, it has become clear that a fourth component exists. This component, which we nickname "Foreground X," is spatially correlated with $100 \mu$ m dust emission, but with a spectrum rising toward lower frequencies in a manner that is incompatible with thermal dust emission.

This fourth component was first discovered in the $C O B E$ Differential Microwave Radiometer (DMR) data by Kogut et al. (1996a, 1996b), who tentatively identified it as free-free emission. It has since been detected in the data sets from Saskatoon (de Oliveira-Costa et al. 1997), Owens Valley Radio Observatory (OVRO; Leitch et al. 1997), the $19 \mathrm{GHz}$ survey (de Oliveira-Costa et al. 1998), and Tenerife (de Oliveira-Costa et al. 1999, hereafter dOC99; Mukherjee et al. 2001). Draine \& Lazarian (1998, hereafter DL98) argued

\footnotetext{
${ }^{1}$ Department of Physics and Astronomy, 209 South 33rd Street, University of Pennsylvania, Philadelphia, PA 19104; angelica@ higgs.hep.upenn.edu.

${ }_{2}^{2}$ Department of Astronomy, Peyton Hall, Ivy Lane, Princeton University, Princeton, NJ 08544.

${ }^{3}$ University of Manchester, Nuffield Radio Astronomy Laboratories, Jodrell Bank, Macclesfield, Cheshire, SK11 9DL, UK.

${ }^{4}$ Instituto de Astrofisica de Canarias, 38200 La Laguna, Tenerife, Spain.

5 Astronomy Department, University of Wisconsin, Madison, WI 53706 .

${ }^{6}$ Mullard Radio Astronomy Observatory, Cavendish Laboratory, Madingley Road, Cambridge, CB3 OHE, UK.

${ }^{7}$ Physics Department, Lewis and Clark College, Portland, OR 97219.
}

against the free-free hypothesis on energetic grounds, and suggested that Foreground $\mathrm{X}$ was caused by dust after all, but via rotational rather than vibrational emission. The fact that the spectrum of Foreground $\mathrm{X}$ is observed to flatten toward lower frequencies and perhaps even turn over around $15 \mathrm{GHz}$ (dOC99) agrees well with the spinning-dust predictions. $^{8}$ Although a consensus has still not been reached on this point (Mukherjee et al. 2001), the case for spinning dust was beginning to look quite solid until recently.

New observations done by Finkbeiner et al. (2001, hereafter F01) have reopened the question about the existence and nature of Foreground X. Using the Green Bank 140 foot telescope, these authors observed 10 IRAS dust filaments at arcminute $\left(\mathrm{FWHM} \sim 6^{\prime}\right)$ scales in the frequencies 5, 8, and $10 \mathrm{GHz} .{ }^{9}$ Although the DL98 spinning-dust model normalized to the Tenerife observations suggested that several filaments should be detected at the $8 \sigma$ level, F01 observations show that only two of these 10 regions are correlated with the $100 \mu \mathrm{m}$ dust map, and only one of these two detections is compatible with the Tenerife results. Moreover, their frequency spectra are consistent with the spinning-dust model of DL98 and inconsistent with freefree emission alone.

The goal of this paper is to perform new cross-correlation tests and to present a model that reconciles these apparently contradictory results. Since the recently completed map from the Wisconsin $\mathrm{H} \alpha$ Mapper (WHAM) should trace freefree emission, it offers a powerful way to test the free-free explanation of Foreground X. A recent cross-correlation analysis of WHAM with QMAP (de Oliveira-Costa et al.

\footnotetext{
${ }^{8}$ Adding information to this puzzle, correlations between dust and $\mathrm{H} \alpha$ maps are marginal (McCullough 1997; Kogut 1997).

${ }^{9}$ The selected regions were carefully chosen to be cold neutral clouds or $\mathrm{H}$ II regions at low density because in both objects, the free-free emission is expected to be subdominant.
} 
2000 , hereafter dOC00) failed to reveal a significant correlation, but this is hardly surprising, since QMAP failed to detect significant contributions of Foreground $\mathrm{X}$ as well. Below, we start out in $\S 2$ by cross-correlating WHAM with the Tenerife data, in which Foreground $\mathrm{X}$ was previously detected at the $4.5 \sigma$ level (dOC99). We find that free-free emission is detected at levels far too low to explain Foreground $X$. In $\S 3$ we therefore proceed to propose an alternative model for what is going on.

\section{CONSTRAINTS FROM THE CROSS-CORRELATION}

\subsection{Cross-Correlation Method}

In this section, we quantify the foreground contamination in the Tenerife data by cross-correlating it with a number of Galactic emission templates. This is done with the multicomponent fit method, described in detail in dOC99. This method models the vector of Tenerife observations $y$ as a sum:

$$
\boldsymbol{y}=\boldsymbol{X a}+\boldsymbol{x}_{\mathrm{CMB}}+\boldsymbol{n},
$$

where $X$ is a matrix whose rows contain the various foreground templates convolved with the Tenerife triple beam (i.e., $\boldsymbol{X}_{i j}$ would be the $i$ th observation if the sky had looked like the $j$ th foreground template), and $a$ is a vector of size $M$ that gives the levels at which these foreground templates are present in the Tenerife data.

The estimate of $\boldsymbol{a}$ is computed by minimizing $\chi^{2} \equiv(y-X a)^{T} C^{-1}(y-X a)$ and by modeling the covariance matrix $\boldsymbol{C}$, including both the experimental noise $\boldsymbol{n}$ and CMB sample variance in the CMB signal $x_{\mathrm{CMB}}$. As in dOC99, our quoted error bars include the effects of both noise and chance alignments between the CMB and the various template maps. The minimum-variance estimate of $a$ is

$$
\hat{a}=\left[X^{T} C^{-1} X\right]^{-1} X^{T} C^{-1} y
$$

with covariance matrix

$$
\boldsymbol{\Sigma} \equiv\left\langle\hat{a} \hat{a}^{T}\right\rangle-\langle\hat{a}\rangle\left\langle\hat{a}^{T}\right\rangle=\left[X^{T} C^{-1} X\right]^{-1}
$$

\subsection{Data Used}

We use the latest version of the Tenerife data (Mukherjee et al. 2001), which has more accurate offset removal than that used in dOC99. To obtain accurate estimates of foreground levels, it is important that the analysis include all relevant emission components and the correlations between them. As a synchrotron template, we use the $408 \mathrm{MHz}$ survey (Haslam et al. 1982, hereafter H82), and the 1420 MHz survey (Reich 1982; Reich \& Reich 1986) for crosschecks. As a template for both vibrational and spinningdust emission, we use the Diffuse Infrared Background Experiment (DIRBE) sky maps at wavelengths from 12 to $240 \mu \mathrm{m}$ (Boggess et al. 1992). Finally, as a tracer of free-free emission, we use the WHAM survey (Haffner et al. 1999). The extent of point-source contamination in the Tenerife data was discussed and estimated in Gutiérrez et al. (2000) and is therefore not addressed in this paper. In practice, we just remove the estimated point-source contribution before calculating the correlations.

\subsection{Cross-Correlation Results}

Cross-correlation results are presented in Table 1 and Figure 1. All fits are done jointly for three templates $(100$

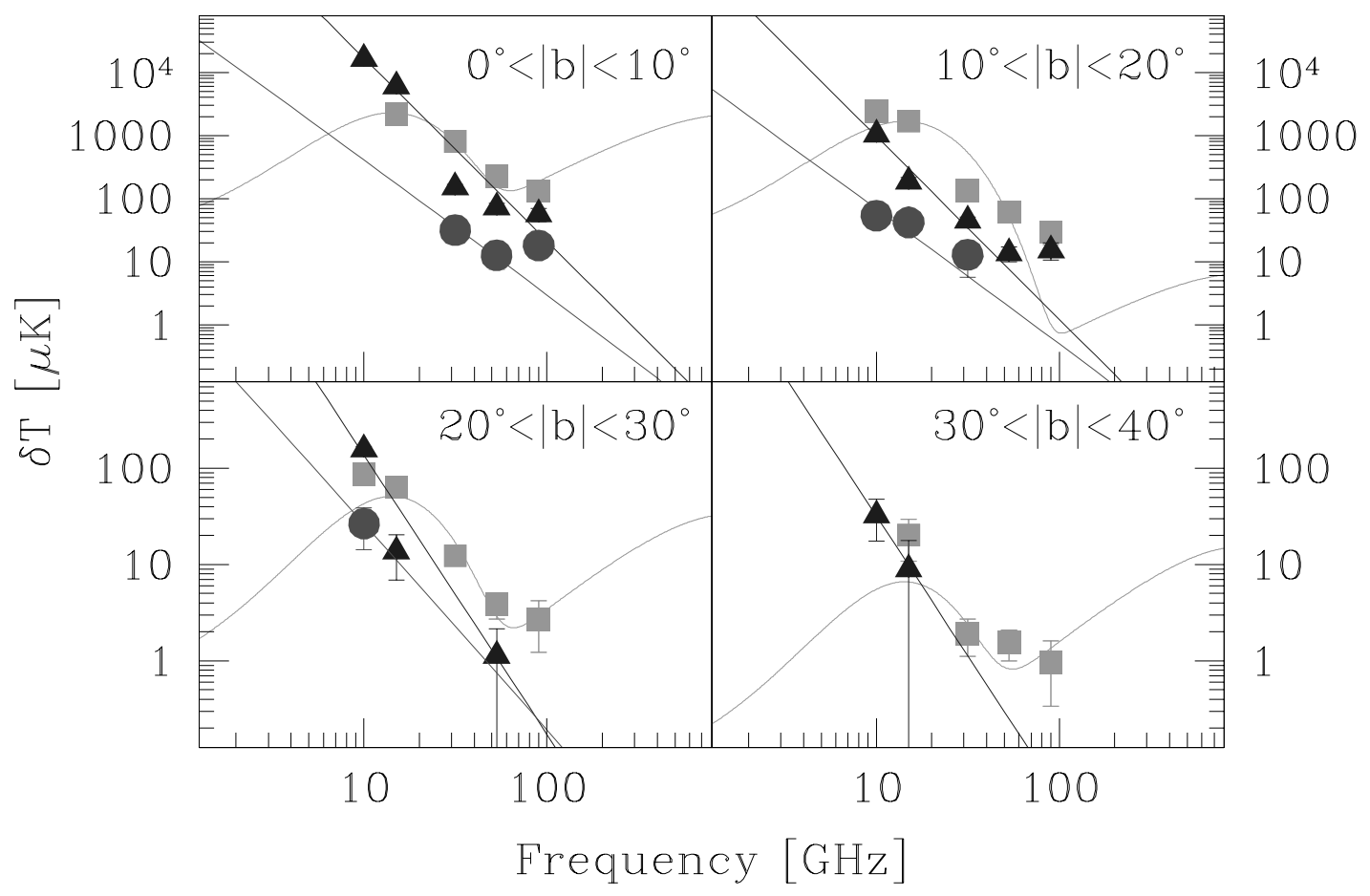

FIG. 1.-Frequency dependence of rms foreground fluctuations, shown for four different Galactic latitude slices. Squares show DIRBE-correlated emission (Foreground X), triangles H82-correlated emission (synchrotron), and circles H $\alpha$-correlated emission (free-free) for four Galactic latitude slices $\left(0^{\circ}-10^{\circ}, 10^{\circ}-20^{\circ}, 20^{\circ}-30^{\circ}\right.$, and $\left.30^{\circ}-40^{\circ}\right)$. The free-free curve is seen to lie below Foreground $\mathrm{X}$ in all cases, typically by about an order of magnitude. Foreground $\mathrm{X}$ therefore cannot be dominated by free-free emission. The corresponding curves represent the best-fit models. The 10 and $15 \mathrm{GHz}$ points are from the Tenerife correlations, and the $31.5,53$, and $90 \mathrm{GHz}$ points are from the DMR correlations. Upper limits are $2 \sigma$. 
TABLE 1

Correlations FOR 10 AND $15 \mathrm{GHz}$ DATA

\begin{tabular}{|c|c|c|c|c|c|}
\hline $\begin{array}{l}\text { Frequency } \\
\qquad(\mathrm{GHz})\end{array}$ & $\mathrm{Map}^{\mathrm{a}}$ & $\hat{\boldsymbol{a}} \pm \delta \hat{\boldsymbol{a}}^{\mathrm{b}}$ & $\hat{\boldsymbol{a}} / \delta \hat{\boldsymbol{a}}$ & $\sigma_{\mathrm{Gal}}$ & $\begin{array}{l}\delta T^{\mathrm{c}} \\
(\mu \mathrm{K})\end{array}$ \\
\hline \multicolumn{6}{|c|}{$|b|>20^{\circ}$} \\
\hline \multirow{3}{*}{$10 \ldots \ldots \ldots$} & $100 \mu \mathrm{m}$ & $71.4 \pm 14.2$ & $5.0^{*}$ & 0.7 & $49.9 \pm 9.9$ \\
\hline & H82 & $71.3 \pm 6.3$ & $11.3^{*}$ & 1.0 & $71.3 \pm 6.3$ \\
\hline & WHAM & $-4.7 \pm 19.9$ & -0.2 & 0.4 & $-1.9 \pm 8.0$ \\
\hline \multirow{3}{*}{$15 \ldots \ldots \ldots$} & $100 \mu \mathrm{m}$ & $51.4 \pm 8.0$ & $6.4^{*}$ & 0.7 & $35.9 \pm 5.6$ \\
\hline & H82 & $4.1 \pm 4.1$ & 1.0 & 1.0 & $4.1 \pm 4.1$ \\
\hline & WHAM & $-13.6 \pm 10.8$ & -1.3 & 0.4 & $-5.4 \pm 4.3$ \\
\hline \multicolumn{6}{|c|}{$|b|>30^{\circ}$} \\
\hline \multirow[t]{3}{*}{$10 \ldots \ldots \ldots$} & $100 \mu \mathrm{m}$ & $-3.5 \pm 40.0$ & -0.1 & 0.2 & $-0.7 \pm 8.0$ \\
\hline & H82 & $5.8 \pm 9.4$ & 0.6 & 0.9 & $5.2 \pm 8.5$ \\
\hline & WHAM & $39.3 \pm 75.2$ & 0.5 & 0.1 & $3.9 \pm 7.5$ \\
\hline \multirow{3}{*}{$15 \ldots \ldots \ldots$} & $100 \mu \mathrm{m}$ & $53.7 \pm 26.3$ & $2.0^{*}$ & 0.2 & $10.7 \pm 5.3$ \\
\hline & H82 & $-4.5 \pm 6.0$ & -0.8 & 0.9 & $-4.0 \pm 5.4$ \\
\hline & WHAM & $-38.1 \pm 43.6$ & -0.9 & 0.1 & $-3.8 \pm 4.3$ \\
\hline \multicolumn{6}{|c|}{$|b| \gtrsim 40^{\circ}$} \\
\hline \multirow[t]{3}{*}{$10 \ldots \ldots \ldots$} & $100 \mu \mathrm{m}$ & $65.9 \pm 60.5$ & 1.1 & 0.2 & $13.2 \pm 12.1$ \\
\hline & H82 & $-4.8 \pm 12.3$ & -0.4 & 0.8 & $-3.8 \pm 9.8$ \\
\hline & WHAM & $19.7 \pm 107.0$ & 0.2 & 0.1 & $1.9 \pm 10.7$ \\
\hline \multirow{3}{*}{$15 \ldots \ldots \ldots$} & $100 \mu \mathrm{m}$ & $27.0 \pm 43.3$ & 0.6 & 0.2 & $5.4 \pm 8.7$ \\
\hline & H82 & $-3.6 \pm 7.5$ & -0.5 & 0.8 & $-2.8 \pm 6.0$ \\
\hline & WHAM & $-55.0 \pm 58.2$ & -0.9 & 0.1 & $-5.5 \pm 5.8$ \\
\hline \multicolumn{6}{|c|}{$|b|>20^{\circ}$} \\
\hline \multirow[t]{3}{*}{$10 \ldots \ldots \ldots$} & "Fink12 $\mu \mathrm{m} "$ & $777.3 \pm 273.8$ & $2.8^{*}$ & 0.03 & $23.3 \pm 8.2$ \\
\hline & H82 & $73.1 \pm 6.3$ & $11.6^{*}$ & 1.0 & $73.1 \pm 6.3$ \\
\hline & WHAM & $5.0 \pm 19.8$ & 0.3 & 0.4 & $2.0 \pm 7.9$ \\
\hline \multirow[t]{3}{*}{$15 \ldots \ldots \ldots$} & "Fink $12 \mu \mathrm{m} "$ & $763.2 \pm 169.7$ & $4.5^{*}$ & 0.03 & $22.9 \pm 5.1$ \\
\hline & H82 & $5.2 \pm 4.1$ & 1.3 & 1.0 & $5.2 \pm 4.1$ \\
\hline & WHAM & $-14.0 \pm 10.9$ & -1.3 & 0.4 & $-5.6 \pm 4.4$ \\
\hline \multicolumn{6}{|c|}{$|b|>20^{\circ}$} \\
\hline \multirow[t]{3}{*}{$10 \ldots \ldots \ldots$} & Combo & $1.0 \pm 0.2$ & $5.0^{*}$ & 58.1 & $58.1 \pm 11.7$ \\
\hline & H82 & $68.5 \pm 6.4$ & $10.7^{*}$ & 1.0 & $68.5 \pm 6.4$ \\
\hline & WHAM & $-15.2 \pm 20.2$ & -0.8 & 0.4 & $-6.1 \pm 8.1$ \\
\hline \multirow[t]{3}{*}{$15 \ldots \ldots \ldots$} & Combo & $1.0 \pm 0.2$ & $5.0^{*}$ & 32.4 & $32.4 \pm 6.5$ \\
\hline & H82 & $4.5 \pm 4.1$ & 1.1 & 1.0 & $4.5 \pm 4.1$ \\
\hline & WHAM & $-13.9 \pm 10.8$ & -1.3 & 0.4 & $-5.6 \pm 4.3$ \\
\hline
\end{tabular}

NoTE.-The asterisks $(*)$ denote statistically significant $(>2 \sigma)$ values.

a The DIRBE, H82, and WHAM correlations listed correspond to a joint 100 $\mu \mathrm{m}-\mathrm{H} 82-\mathrm{H} \alpha$ fit.

${ }^{\mathrm{b}}$ The term $\hat{\boldsymbol{a}}$ has units of $\mu \mathrm{K} \mathrm{MJy}^{-1} \mathrm{sr}^{-1}$ for the $100 \mu \mathrm{m}, \mu \mathrm{K} \mathrm{K} \mathrm{K}^{-1}$ for the H82, and $\mu \mathrm{K} \mathrm{R}^{-1}$ for the WHAM template.

c The term $\delta T=(\hat{\boldsymbol{a}} \pm \delta \hat{\boldsymbol{a}}) \sigma_{\mathrm{Gal}}$.

$\mu \mathrm{m}$; H82; WHAM), and statistically significant (greater than $2 \sigma$ ) correlations listed in Table 1 are marked by asterisks. Since the fluctuation levels depend strongly on Galactic latitude, we perform our analysis for six different latitude slices of roughly equal area (Fig. 1), as well as for three different latitude cuts (Table 1): $20^{\circ}, 30^{\circ}$, and the "Tenerife cut" (which consists of data inside the region $160^{\circ}<$ R.A. $<250^{\circ}$, corresponding to Galactic latitudes $|b| \gtrsim 40^{\circ}$ ). For definiteness, we use the DIRBE $100 \mu \mathrm{m}$ channel when placing all limits shown in this subsection, since it is the least noisy of the DIRBE channels, and the H82 map, since it is the synchrotron template at lowest frequency.

Figure 1 shows the corresponding fluctuations in antenna temperature in the Tenerife data $\left(\delta T=\hat{\boldsymbol{a}} \sigma_{\mathrm{Gal}}\right.$, where $\sigma_{\mathrm{Gal}}$ is the standard deviation in the template map). The H82 and DIRBE correlations are seen to be consistent with those from dOC99. Synchrotron emission (triangles) generally dominates the rms foreground fluctuations at $10 \mathrm{GHz}$. At $15 \mathrm{GHz}$, on the other hand, Foreground $\mathrm{X}$ (squares) is seen to dominate, except in the Galactic plane itself. The key new result here is the inclusion of the WHAM data, showing that free-free emission (circles) is about an order of magnitude below Foreground $X$ over the entire range of frequencies and latitudes where it is detected. This means that Foreground $\mathrm{X}$ cannot be explained by free-free emission. The corresponding values of $a_{i}$ (in units of $\mu \mathrm{K} \mathrm{R}^{-1}$ ) are consistent with gas at $8000 \mathrm{~K}$ (Bennett et al. 1992) for lati- 
TABLE 2

$100 \mu \mathrm{m}-\mathrm{H} \alpha$ CORRELATIONS

\begin{tabular}{|c|c|c|c|}
\hline Patch $^{a}$ & $\begin{array}{c}\hat{a} \pm \delta \hat{a} \\
\left(\mathrm{RJy}^{-1} \mathrm{Sr}^{-1}\right)\end{array}$ & $\hat{a} / \delta \hat{a}$ & References \\
\hline$b=-65^{\circ}$. & $0.79 \pm 0.24$ & $3.3^{*}$ & 1 \\
\hline$b=-21^{\circ} \ldots$ & $0.85 \pm 0.44$ & 1.9 & 2 \\
\hline$b=27^{\circ} \ldots \ldots$ & $0.34 \pm 0.33$ & 1.0 & 2 \\
\hline$b=27^{\circ}$. & $0.25 \pm 0.19$ & 1.3 & 3 \\
\hline$|b|>20^{\circ} \ldots$ & $0.18 \pm 0.07$ & $2.6^{*}$ & 4 \\
\hline$|b|>30^{\circ}$ & $0.17 \pm 0.04$ & $4.3^{*}$ & 4 \\
\hline Tenerife cut ............ & $0.04 \pm 0.03$ & 1.3 & 4 \\
\hline $00^{\circ} \lesssim|b| \lesssim 10^{\circ} \ldots \ldots$ & $0.004 \pm 0.100$ & 0.04 & 4 \\
\hline $10^{\circ} \lesssim|b| \lesssim 20^{\circ} \ldots \ldots$ & $0.04 \pm 0.17$ & 0.2 & 4 \\
\hline $20^{\circ} \lesssim|b| \lesssim 30^{\circ} \ldots \ldots$ & $0.16 \pm 0.15$ & 1.1 & 4 \\
\hline $30^{\circ} \lesssim|b| \lesssim 42^{\circ} \ldots \ldots$ & $0.16 \pm 0.03$ & $5.3^{*}$ & 4 \\
\hline $42^{\circ} \lesssim|b| \lesssim 56^{\circ} \ldots \ldots$ & $0.14 \pm 0.10$ & 1.4 & 4 \\
\hline $56^{\circ} \lesssim|b| \lesssim 90^{\circ} \ldots \ldots$ & $0.14 \pm 0.08$ & 1.8 & 4 \\
\hline
\end{tabular}

NotE.-The asterisks $\left({ }^{*}\right)$ denote statistically significant $(>2 \sigma)$ values.

${ }^{\text {a }}$ Patches are centered at the indicated $b$ coordinates.

REFERENCES.-(1) McCullough 1997; (2) Kogut 1997; (3) dOC00; (4) this work, for the $15 \mathrm{GHz}$ data.

tudes $|b|=0^{\circ}-10^{\circ}$ and frequencies $\geq 30 \mathrm{GHz}$, with substantial scatter elsewhere. ${ }^{10}$

As in de Oliveira-Costa et al. $(1997,1998)$, dOC99, and dOC00, the cross-correlation software was tested by analyzing constrained realizations of CMB and Tenerife instrument noise. From 1000 realizations, we recovered unbiased estimates $\hat{\boldsymbol{a}}$ with a variance in excellent agreement with equation (3). As an additional test, we computed $\chi^{2} \equiv(\boldsymbol{y}-\boldsymbol{X a})^{T} \boldsymbol{C}^{-1}(\boldsymbol{y}-\boldsymbol{X a})$ and obtained $\chi^{2} / N \approx 1$ in almost all cases.

We also performed a joint fit retaining the $100 \mu \mathrm{m}, \mathrm{H} 82$, and WHAM templates in the Tenerife observing region, but replacing the Tenerife data by $C O B E$ DMR data at $31.5,53$, and $90 \mathrm{GHz}$. These results are also plotted in Figure 1 by multiplying the measured coefficients $\hat{a}$ by the template rms $\sigma_{\mathrm{Gal}}$ corresponding to the Tenerife triple beam, and they show that our conclusions extend to higher frequencies as well. ${ }^{11}$

Since we find substantially lower levels of free-free emission than Foreground X, the corresponding templates $(100$ $\mu \mathrm{m}$ and WHAM) cannot be very highly correlated. We confirmed this by a direct cross-correlation analysis between these two templates, as seen through the Tenerife triple beam in the Tenerife observing region, and found the dimensionless correlation coefficient between the two maps to be in the range $5 \%-30 \%$, depending on Galactic latitude. The corresponding results in physical units $\left(\mathrm{R} \mathrm{MJy}^{-1} \mathrm{sr}^{-1}\right)$ are shown in Table 2 . Since the statistical properties of these maps are not accurately known, we estimated the error bars

\footnotetext{
${ }^{10}$ Although the cross-correlation technique can accurately determine the dominant components, the detailed results for strongly subdominant components must be taken with a grain of salt. This is because any complications with the dominant components (e.g., slight spatial variations in their frequency dependence) that are not included in the model of eq. (1) will act as excess noise on the remaining components. Although the analysis clearly demonstrates that free-free emission is subdominant, the formal error bars on this component are therefore likely to be smaller than the true errors.

${ }^{11}$ A full DMR-WHAM correlation analysis is in progress (A. Kogut et al., in preparation), so the results presented here should be viewed as preliminary. For all-sky cross-correlations, large-scale variations in the WHAM map will need to be better accounted for.
}

by repeating the analysis with one of the templates replaced by $4 \times 36=144$ transformed maps, rotated around the Galactic axis by multiples of $10^{\circ}$ and/or flipped vertically and/or horizontally. Significant correlations are found only for the $b>20^{\circ}$ and $b>30^{\circ}$ cuts, and the bulk of this correlation seems to come from a region of the sky with $30^{\circ}<b<42^{\circ}$.

For comparison, see the McCullough (1997), Kogut (1997), and dOC00 results shown in Table 2. This table indicates significant variations across the sky in the relationship between $\mathrm{H} \alpha$ and the $100 \mu \mathrm{m}$ emission, which could be related to variations in the hydrogen ionization fraction.

Finally, writing the frequency dependence as $a \propto v^{\beta}$ and recalling that the correlation coefficient is, by definition, $a=1 \mathrm{~K} / \mu \mathrm{K}=10^{6}$ for $\mathrm{H} 82$ at $408 \mathrm{MHz}$, we obtain the spectral index limits of $-2.9 \lesssim \beta \lesssim-3.6$ for the $10 \mathrm{GHz}-\mathrm{H} 82$ correlation and $-3.2 \lesssim \beta \lesssim-3.7$ for the $15 \mathrm{GHz}-\mathrm{H} 82$ correlation. These values are slightly steeper than the canonical sub-GHz slope of $-2.7 \lesssim \beta \lesssim-2.9$ (Davies, Watson, \& Gutiérrez 1996; Platania et al. 1998), but consistent with a steepening of the spectrum of cosmic-ray electrons at higher energies (Rybicki \& Lightman 1979). The relatively high Tenerife synchrotron signal seen in Table 1 could be interpreted as a slight spatial variability of the frequency dependence (Tegmark et al. 2000).

\subsection{Power Spectrum of Foregrounds}

Figure 1 shows the rms contribution of each foreground. In order to understand which angular scales contribute most to this rms, we compute the angular power spectra of the template emissions. The angular power spectra of the DIRBE, H82, and WHAM components are shown in Figure 2 for the $20^{\circ}-30^{\circ}$ slice of Figure 1 , scaled to $15 \mathrm{GHz}$ using the correlation coefficients found above. All three power spectra are seen to be compatible with a power law of

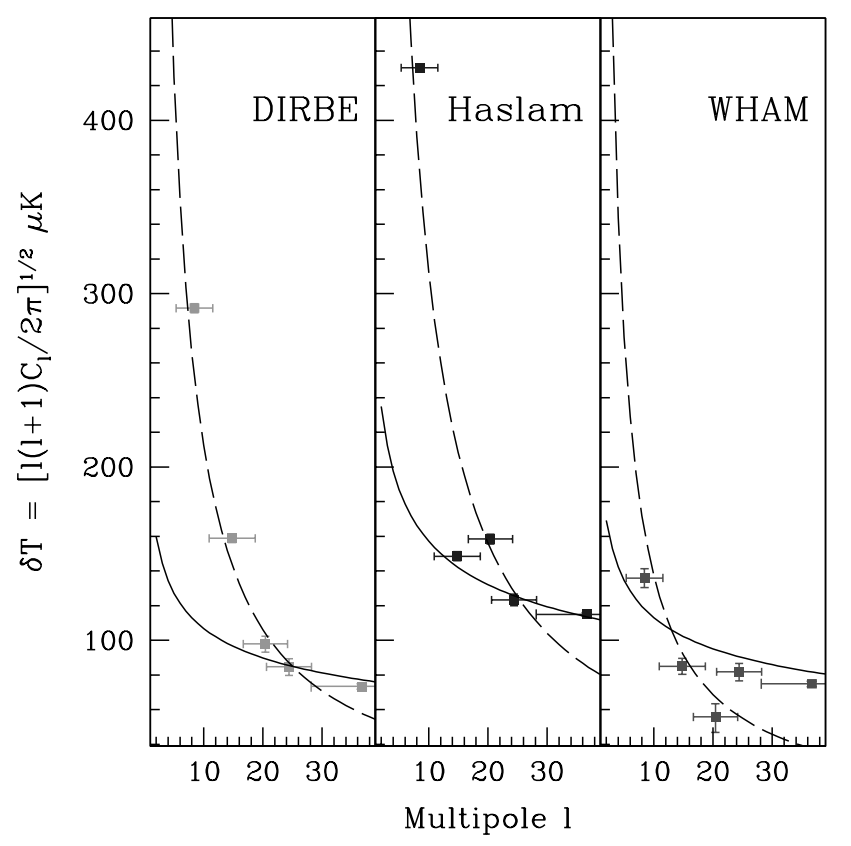

FIG. 2.-Angular power spectra for the DIRBE, H82, and WHAM components, as seen in the Tenerife data. Results are from the $20^{\circ}-30^{\circ}$ slice shown in Fig. 1. The curves show $\ell^{-6}$ (dashed line) and $\ell^{-3}$ (solid line) power laws. 
$\ell^{-3}$ (solid line) at small angular scales $(\ell>10-15),{ }^{12}$ while DIRBE and H82 are seen to steepen further on very large scales $(\ell<10-15)$ to a power law closer to $\ell^{-6}$ (dashed line).

We remind the reader that there is, strictly speaking, no such thing as the power spectrum of a Galactic foreground, since the latitude dependence implies that it is not an isotropic random field. What we refer to here as the power spectrum of a foreground is simply the quantity that we care about in practice: its contribution to the measurement of a CMB power spectrum. We have therefore computed the curves in Figure 2 by convolving the template maps with the Tenerife triple beam and have treated the result as if it were a CMB map, computing quadratic band power estimators as described in Tegmark (1997) and Bond, Jaffe, \& Knox (1998).

\section{AN ALTERNATIVE MODEL FOR FOREGROUND $X$}

The results presented above show that free-free emission cannot explain Foreground X. Since there is currently only one other strong contender, spinning dust as proposed by DL98, the case would appear to be closed..$^{13}$ However, as we describe below, the situation seems to be a little more complicated.

Correlations at $100 \mu \mathrm{m}$ have been detected at high significance on large $\left(3^{\circ}-7^{\circ}\right)$ angular scales: at $4.2 \sigma$ at $31 \mathrm{GHz}$ by the DMR, at $6.6 \sigma$ in the $19 \mathrm{GHz}$ survey, at $6.4 \sigma$ at $15 \mathrm{GHz}$ by Tenerife, and at $5.1 \sigma$ at $10 \mathrm{GHz}$ by Tenerife. These detections reveal a spectrum rising toward lower frequencies, with hints of a plateau or turnover between 10 and $15 \mathrm{GHz}$. On intermediate (degree) scales, the Saskatoon data gave a marginal detection while the QMAP data gave only an upper limit. At small (arcminute) scales, the situation is even more confusing: although F01 recently reported a correlation of an $\mathrm{H}$ II region (LPH $201.663+1.643$ ) and a dark cloud (L1622) at 5, 8, and $10 \mathrm{GHz}$ with the SFD98 $100 \mu \mathrm{m}$ map (Schlegel, Finkbeiner, \& Davis 1998), these values are not in concordance with the originally reported

\footnotetext{
12 Our DIRBE result is compatible with that reported by Wright (1998).

${ }^{13}$ There is also the possibility that Foreground $\mathrm{X}$ is due to magnetic dipole emission from ferromagnetic grain materials (Draine \& Lazarian 1999). This model can be ruled out if Foreground $X$ can be shown to correlate better with $12 \mu \mathrm{m}$ than with $100 \mu \mathrm{m}$ emission.
}

value given by OVRO at $14.5 \mathrm{GHz}$ (Leitch et al. 1997). ${ }^{14}$ Moreover, only the dark cloud correlation detected by F01 seems to be clearly consistent with our (large-scale) Tenerife result of $\hat{a}=71.4 \pm 14.2 \mu \mathrm{K} \mathrm{MJy}^{-1} \mathrm{sr}^{-1}$ (see Table 1). This discrepancy cannot be blamed on variations in dust column density between different sky patches, since they would not affect the dust-to-CMB conversion factor $\hat{a}$. In summary, on average the most solid detections of Foreground $\mathrm{X}$ are all on scales of a few degrees or larger. Why is this foreground so elusive on smaller scales?

\subsection{A Grain-Size Segregation Model}

Here we argue in support of a model in which Foreground $\mathrm{X}$ is spinning dust after all, and the problem is with the template used to find it, i.e., $100 \mu \mathrm{m}$ dust emission.

Galactic dust grains come in a wide range of sizes (Weingartner \& Draine 2001a). The $100 \mu \mathrm{m}$ template is dominated by large dust grains that are close to thermal equilibrium with the interstellar radiation field and are radiating thermally at temperatures around $20 \mathrm{~K}$ (Reach et al. 1995). In contrast, the small grains that can spin fast enough to produce the rotational emission of DL98 cool faster than the mean time between two photon absorption events. Therefore, they spend most of their time near their ground state. They get heated to as much as a few hundred kelvin when they absorb a photon, and radiate nonthermally with a spectrum that is both bluer and broader than that for large thermalized grains (Draine \& Lee 1984). As a result, the emission from small grains peaks at shorter wavelengths, such as $10-30 \mu \mathrm{m}$. Indeed, it is such nonequilibrium behavior that enables the DL98 emission mechanism to work.

Previous work has generally assumed that the relative abundance of grain sizes is independent of position and that the $100 \mu \mathrm{m}$ map (dominated by large grains) is a good tracer for small grains as well. However, this seems not to be the case. A simple visual comparison of the IRAS maps at different frequencies suggests that although the large-scale features generally match up, small-scale features can be quite different (see Fig. 3).

Therefore, our proposed solution to the spinning-dust

\footnotetext{
${ }^{14}$ The statistical significance of this result is unclear, since the OVRO measurement was published without error bars.
}
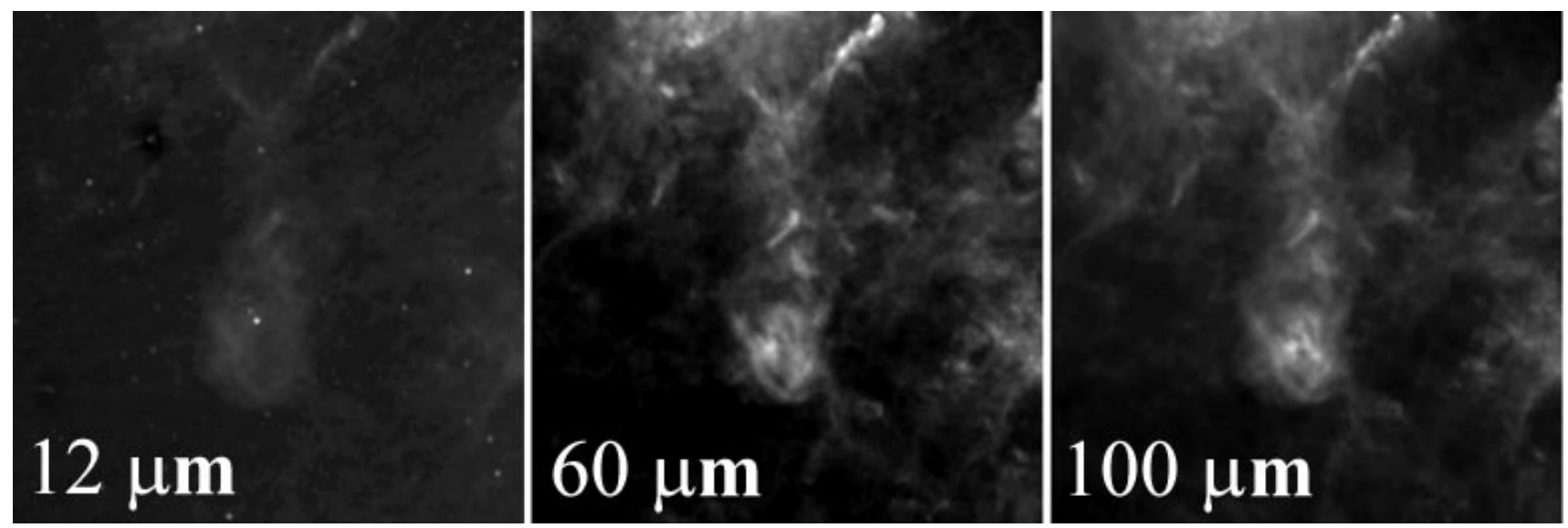

FIG. 3. - IRAS images at 12,60 , and $100 \mu \mathrm{m}$ from the "mushroom" cloud MBM 20. These images are squares of $12^{\circ} .8 \times 12.8$ centered at $l=210^{\circ} .9$, $b=-34.5$. A simple visual comparison of these IRAS images at different frequencies suggests that although the large-scale features generally match up, small-scale features can be quite different. Experiments such as ground-based interferometers, MAP, and Planck LFI may have the angular resolution at the relevant low frequencies that are needed to be able to confirm or to rule out our grain-segregation model. 


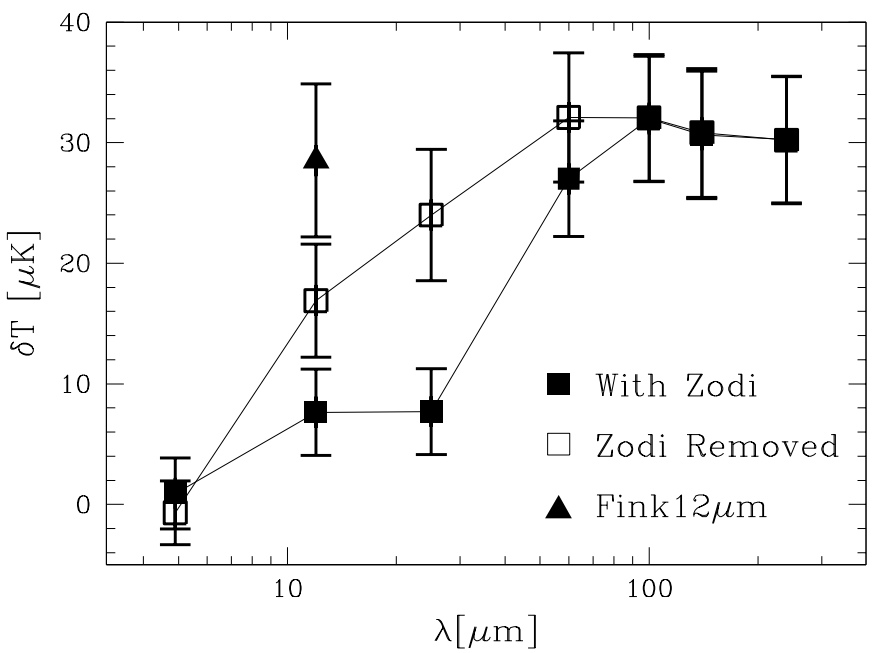

Fig. 4.-Dust correlations for the $12-240 \mu \mathrm{m}$ DIRBE maps at $15 \mathrm{GHz}$. Correlations of DIRBE maps with zodiacal emission are shown by filed squares, correlations of DIRBE maps with zodiacal emission removed are shown by open squares, and the correlation with the $12 \mu \mathrm{m}$ map with zodiacal emission and point sources removed is shown by a filled triangle.

puzzle is that this component (small grains) correlates well with $100 \mu \mathrm{m}$ emission (large grains) only on fairly large angular scales. Occasional agreements on small scales would of course not be precluded (which could explain the F01 and Leitch et al. 1997 results), but should not be expected to hold in general.

As pointed out by Weingartner \& Draine (2001b), it is not physically implausible for small and large dust grains to be separated. They showed that small dust grains can stay locked to the gas, while large dust grains in diffuse clouds can drift because of the effects of anisotropic starlight. After $10^{7} \mathrm{yr}$, a typical lifetime of a diffuse interstellar cloud, such drifts can separate large and small grains by up to a few degrees at high Galactic latitudes.

\subsection{Testing our Model}

A first prediction of this model is that shorter wavelength dust maps should trace spinning dust at least as well as a $100 \mu \mathrm{m}$ map (indeed, better on small scales). ${ }^{15}$ To test this, we repeated our analysis with the $100 \mu \mathrm{m}$ map replaced by DIRBE maps at other wavelengths. The results are shown in Figure 4 (bottom curve). At first glance, it appears inconsistent with our prediction. However, contributions other than Galactic dust can clearly spoil the correlation, and several such contaminants are known to be present. Indeed, the figure shows that removing zodiacal emission from dust in our solar system increases the short wavelength correlations so much that the $60 \mu \mathrm{m}$ map traces spinning dust marginally better than the $100 \mu \mathrm{m}$ map (top curve). The shorter wavelength maps also contain a substantial pointsource contribution. We used a new merged and destriped DIRBE $+I R A S 12 \mu \mathrm{m}$ map $^{16}$ to eliminate all $5 \sigma$ point sources before convolving it with the Tenerife beam. Figure 4 shows that this new $12 \mu \mathrm{m}$ template ("Fink $12 \mu \mathrm{m}$ ") traces spinning dust almost as well as the $100 \mu \mathrm{m}$ map. For

\footnotetext{
15 This prediction was also made by DL98, who argued that the 30 $\mathrm{GHz}$ emission and diffuse $12 \mu \mathrm{m}$ emission should be correlated, since both originate in grains containing $\approx 100$ atoms.

${ }_{16}$ This new map was constructed in the same fashion as the SFD98 100 $\mu \mathrm{m}$ map (for details about how such composite maps are made, see Schlegel et al. 1998 or http://www.astro.princeton.edu/ schlegel/dust/ index.html).
}

comparison, correlations with the $12 \mu \mathrm{m}$ map are shown in the bottom of Table 1 .

We tried two additional approaches to further increase the correlations (neither of which helped more than marginally). The first was to apply a zodiacal cut on the zodiacal-emission-removed maps. The fact that this failed to increase correlations suggests that the zodiacal-emission removal performed by the DIRBE team was already adequate for our purposes. The second was to compute the linear combination of all DIRBE maps that gave the best correlation to see if contaminants could be identified and subtracted spectrally. Correlations with this composite map are also shown in the bottom of Table 1 (see "Combo"). Our interpretation of these negative results is that our large dust grain templates are already tracing spinning dust quite well on large scales, so that it is impossible to do much better. Rather, the key tests will involve the correlation on small angular scales.

\section{DISCUSSION}

We have used the WHAM map to show that DIRBEcorrelated microwave emission (Foreground $\mathrm{X}$ ) cannot be explained as free-free emission. Since the spinning-dust alternative has been challenged by recent small-scale observations, we have argued for an alternative model involving small-scale grain segregation, in which small and large dust grains are well correlated only on large angular scales.

We found that the zodiacal-emission-cleaned $60 \mu \mathrm{m}$ DIRBE map traces Foreground X marginally better than the $100 \mu \mathrm{m}$ map, and that even the $12 \mu \mathrm{m}$ map does a good job after zodiacal-emission and point-source removal. Although these preliminary results are mildly encouraging, the smoking-gun test of this model will involve crosscorrelating dust maps at various frequencies with highresolution CMB data in the $10-30 \mathrm{GHz}$ range.

Fortunately, such maps should be available shortly from CMB experiments such as ground-based interferometers, the NASA MAP satellite, ${ }^{17}$ and the Planck LFI. ${ }^{18}$ For instance, the expected sensitivity of the MAP $22 \mathrm{GHz}$ channel is about $11 \mu \mathrm{K}$ per $56^{\prime}$ pixel. For comparison, the expected small-scale fluctuations from spinning dust at 22 $\mathrm{GHz}$ are of the order of $20 \mu \mathrm{K}$ at high Galactic latitudes, ranging from a few to $10^{2} \mu \mathrm{K}$ from a clean to a dirty region. ${ }^{19}$ Since $M A P$ will measure tens of thousands of such pixels, it should be readily able to confirm or rule out our model. The prediction is that the small-scale signal will be substantially better traced by shorter wavelength dust maps. Since various contaminants may be important in these short-wavelength maps (e.g., polycyclic aromatic hydrocarbons), it will also be worth performing a multicomponent fit using dust maps at all available frequencies to find the linear combination of dust maps that constitutes the best spinning-dust template. High-resolution lowfrequency ground-based experiments such as $\mathrm{CBI}^{20}$ and DASI $^{21}$ may also be able to test our hypothesis, but this is

\footnotetext{
${ }_{17}$ Available at http://map.gsfc.nasa.gov.

${ }^{18}$ Available at http://astro.estec.esa.nl/SA-general/Projects/Planck.

${ }^{19}$ We made this estimate by high-pass filtering the "Fink $12 \mu \mathrm{m}$ " map to the degree scale; this was done by taking the difference of two "Fink $12 \mu \mathrm{m}$ " maps, one smoothed by 0.7 and another by $2^{\circ}$. Using $\hat{\boldsymbol{a}} \approx$ $760 \mu \mathrm{m} \mathrm{MJy}^{-1} \mathrm{sr}^{-1}$ from Table 1, we converted this difference map to $\mu \mathrm{K}$, and finally made a small spectral correction from 15 to $22 \mathrm{GHz}$.

${ }^{20}$ Available at http://astro.caltech.edu/ tjp/CBI.

${ }^{21}$ Available at http://astro.uchicago.edu/dasi
} 
far from clear, since they operate in the $\mathrm{Ka}$ band (26-36 $\mathrm{GHz}$ ), in which the spinning-dust signal is small. In conclusion, we have proposed a resolution to the puzzle of Foreground $\mathrm{X}$. Observations during the coming year should be able to test it.

We would like to thank Bruce Draine for useful com- ments. Support was provided by NASA grants NAG 5-9194 (né NAG 5-6034) and NAG 5-11099, the University of Pennsylvania Research Foundation, and the David and Lucille Packard Foundation. WHAM is supported by the NSF through grant AST 96-19424. We acknowledge the NASA office of Space Sciences, the COBE flight team, and all those who helped process and analyze the DIRBE data.
Bennett, C. L., et al. 1992, ApJ, 396, L7

Boggess, N. W., et al. 1992, ApJ, 397, 420

Bond, J. R., Jaffe, A. H., \& Knox, L. E. 1998, Phys. Rev. D, 57, 2117

Davies, R. D., Watson, R. A., \& Gutiérrez, C. M. 1996, MNRAS, 278, 925

de Oliveira-Costa, A., et al. 1997, ApJ, 482, L17 .1998, ApJ, 509, L9 1999, ApJ, 527, L9 (dOC99) .2000, ApJ, 542, L5 (dOC00)

Draine, B. T., \& Lazarian, A. 1998, ApJ, 494, L19 (DL98) 1999, ApJ $, 512,740$

Draine, B. T., \& Lee, H. M. 1984, ApJ, 285, 89

Finkbeiner, D. P., Davis, M., Frank, C., Schlegel, D. J., \& Heiles, C. 2001, ApJ, in press (F01)

Gutiérrez, C. M., et al. 2000, ApJ, 529, 47

Haffner, L. M., et al. 1999, ApJ, 523, 223

Haslam, C. G. T., et al. 1982, A\&AS, 47, 1 (H82)

Kogut, A. 1997, AJ, 114, 1127

Kogut, A., et al. 1996a, ApJ, 460, 1

\section{REFERENCES}

Kogut, A., et al. 1996b, ApJ, 464, L5

Leitch, E. M., et al. 1997, ApJ, 486, L23

McCullough, P. R. 1997, AJ, 113, 2186

Mukherjee, P., Jones, A. W., Kneissl, R., \& Lasenby, A. N. 2001, MNRAS, 320,224

Platania, P., et al. 1998, ApJ, 505, 473

Reach, W. T., et al. 1995, ApJ, 451, 188

Reich, P., \& Reich, W. 1986, A\&AS, 63, 205

Reich, W. 1982, A\&AS, 48, 219

Rybicki, G. B., \& Lightman, A. P. 1979, Radiative Processes in Astrophysics (New York: Wiley)

Schlegel, D. J., Finkbeiner, D. P., \& Davis, M. 1998, ApJ, 500, 525 (SFD98)

Tegmark, M. 1997, Phys. Rev. D, 56, 4514

Tegmark, M., et al. 2000, ApJ, 530, 133

Weingartner, J. C., \& Draine, B. T. 2001a, ApJ, 548, 296 2001b, ApJ, 553, 581

Wright, E. L. 1998, ApJ, 496, 1 